\title{
Predicting Neovascular Glaucoma risk in a Chinese Proliferative Diabetic Retinopathy population: development and assessment of a new predictive nomogram
}

\author{
Xinyue Zhang \\ Shengjing Hospital of China Medical University \\ li xu( $\nabla$ xu-li1149@163.com ) \\ Shenyang the fourth hospital https://orcid.org/0000-0003-0421-253X \\ Xiaolong Chen \\ Shengjing Hospital of China Medical University
}

\section{Research article}

Keywords: Neovascular glaucoma(NVG),Proliferative diabetic retinopathy(PDR),Predictors, Nomogram

Posted Date: September 11th, 2020

DOI: https://doi.org/10.21203/rs.3.rs-30623/v2

License: (c) (1) This work is licensed under a Creative Commons Attribution 4.0 International License.

Read Full License 


\section{Abstract}

Background: The aim of this study was to develop and evaluate a postoperative NVG risk nomogram based on the clinical data of a Chinese population of patients with PDR.

Methods: A prediction model has been established based on the clinical data of 107 PDR patients who underwent vitrectomy from March,2017 to March,2018 in Shenyang Fourth People's Hospital, and they were followed up for at least 12 months. The presence or absence of NVG were observed.The least absolute shrinkage and selection operator regression model was used to optimize feature selection for the postoperative NVG risk model. Multivariable logistic regression analysis was applied to build a predicting model incorporating the feature selected in the least absolute shrinkage and selection operator regression model. C-index, calibration plot, and decision curve analysis were also introduced to evaluate the model. The bootstrapping validation was also utilized to accomplish internal validation.

Results: Risk factors screened out by the model included HbAlc level, presence of diabetic nephropathy and anti-VEGF therapy. The model was testified with a satisfying C-index of 0.852 (95\% Cl: $0.740-$ 0.964).Decision curve analysis showed that the NVG nomogram was clinically useful when intervention was adopted with the NVG possibility threshold of $2 \%$.

Conclusion: This novel nomogram revealed that a good control of HbAlc level, absence of diabetic nephropathy and anti-VEGF therapy are prophylactic factors of postoperative NVG in PDR patients.

\section{Background}

Neovascular glaucoma (NVG) secondary to proliferative diabetic retinopathy (PDR) is a potentially devastating condition, which is often refractory to medical treatment[1]. When the affected eye becomes completely blind, the patient usually complains of excruciating pain, which might lead to ocular enucleation in the end.The condition was firstly described in 1871, where neovascularization of iris and anterior chamber angle was triggered by retinal ischemia,subsequently leading to the formation of fibrous tissue proliferation and synechial angle closure.As a result,elevated intraocular pressure(IOP) and severe visual impairment are found.

Current treatment options mainly include anti-vascular endothelial growth factor (VEGF) medications,panretinal photocoagulation(PRP) and surgical methods,such as trabeculectomy,implantation of aqueous drainage devices,Nd-Yag cyclophotocoagulation,vitrectomy with PRP and trabeculectomy and cyclocryotherapy[2].The gist of theses various treatments is to treat both the elevated IOP and the underlying abnormalities immediately.However,the therapeutic effect usually varies with different individuals because of delayed diagnosis or poor management.

Previous research has failed to reveal the pathogenesis and prognostic factors of postoperative NVG secondary to PDR.This paper focus on detecting some related clinical factors by developing a credible 
but simple prediction tool for PDR patient group who underwent vitrectomy to assess the risk of NVG based on clinical data in their treating process.

\section{Methods}

\section{Patients}

Our study was approved by Ethics Committee of our hospital and adhered to the tenets of Helsinki Declarations.Informed consents were obtained from all the participants.Patients were retrospectively collected in the Ophthalmology Department of Shenyang Fourth People Hospital from March,2017 to March,2018 and followed up for at least 12 months. They were included if they were diagnosed as PDR and underwent vitrectomy in our hospital.All the vitrectomy procedures were accomplished by the same experienced ophthalmologist using a 23-gauge three-port vitrectomy system(Alcon Laboratories,Inc.).NVG was defined as neovascularization of the iris and/or iridocorneal angle, with a history of IOP of more than

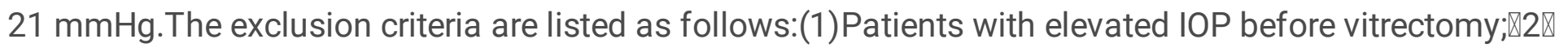
Patients who accepted intravitreal injection of Triamcinolone;(3)Patients with recurrent vitreous hemorrhage.

\section{Data Collection}

The medical records and surgical procedures of eligible patients were retrospectively reviewed.Subject characteristics examined included age,gender,type of diabetic mellitus(DM),HbAlc(glycosylated hemoglobin), diabetic nephropathy(DN),hyperlipemia, cardiovascular diseases,smoking and alcoholism.Surgical procedures included anti-VEGF therapy,history of PRP(within 3 months),status of lens,vitreous tamponade,internal limiting membrane区ILM $₫$ peeling,vitreous hemorrhage $(\mathrm{VH})$ and tractional retinal detachment(RD).And the presence or absence of NVG were noted.

\section{Statistical Analyses}

All data including systemetic and ocular characteristics which are categorical variables are displayed in numbers and percentages. Statistical analysis was performed using the R software (Version 3.1.1; https://www.R-project.org).

We performed least absolute shrinkage and selection operator (LASSO) to remove redundancy within the systemetic and ocular information by selecting the most prognostic characteristics in predicting the prevalence of postoperative NVG among PDR group.LASSO is a more reliable method to select some prognostic features in clinical data and information, which was originally used in

oncology[3-4].So in our study,we firstly used this method to screen out the optimal predictive features in risk factors from the PDR patients with postoperative NVG. We also conducted multivariate analysis to identify the risk factors of postoperative NVG in PDR patients who underwent vitrectomy, and the odds ratio (OR) with $95 \%$ confidence interval $(95 \% \mathrm{Cl})$ of each related factor was displayed. Moreover,some risk factors that were considered to be clinically meaningful were also included[5-6]. Nomograms were built 
on the basis of the clinical factors screened out by Lasso regression and multivariate analysis to construct the clinical integration model[7-8]. A nomogram can provide an individualized, evidence-based, highly accurate risk estimation which facilitates decision making.

The bootstrapped calibration curves demonstrate the difference between a prediction and observation. [9].So overall $\mathrm{C}$ index(concordance index) has been introduced as a parameter to describe the performance of a given model applied to the population under consideration and discuss the statistic used as its sample estimate[10].Decision curve analysis is based on the principle that the relative harms of false positives and false negatives can be expressed in terms of a probability threshold.[11].The net benefit was calculated by subtracting the proportion of all patients who are false positive from the proportion of the patients who are true positive.So it could model the clinical consequences of true and false positives in order to assess clinical relevance[12].

\section{Results}

\section{Patients' Baseline Characteristics}

A total of 107 patients who underwent vitrectomy for PDR in our hospital were enrolled(52 males and 55 females;mean age $55.24 \pm 14.31$ years[range $22-78$ years]). The mean course of diabetic mellitus was $9.7 \pm 3.8$ years[range 2.5 to 21 years].Among them,18(16.8\%) developed NVG during 12-month follow up postoperatively.So they were divided into NVG and non-NVG group.All data of patients including demographic, disease, and treatment features in the two groups are given in Table 1.

Table 1 Differences between demographic and clinical characteristics of NVG and non-NVG groups 


\begin{tabular}{|c|c|c|c|}
\hline \multirow[t]{2}{*}{ Characteristics } & \multicolumn{3}{|c|}{$\mathrm{n}(\%)$} \\
\hline & \multicolumn{3}{|c|}{$\overline{\text { NVG}}[\mathrm{n}=18 \square$ non-NVG $n=89 \square$ Total $(\mathrm{n}=107)$} \\
\hline \multicolumn{4}{|l|}{ Age } \\
\hline$<=50$ & $7(38.89)$ & $26 \square 29.21 \square$ & $33 \square 30.84 \square$ \\
\hline $51-59$ & $2(11.11)$ & $20 \square 22.47 \square$ & $22 \llbracket 20.56 \square$ \\
\hline $60-69$ & $7(38.89)$ & $36 \square 40.45 \square$ & $43 \square 40.19 \square$ \\
\hline$>=70$ & $2(11.11)$ & 7ロ7.87ロ & $9 \square 8.41 \square$ \\
\hline \multicolumn{4}{|l|}{ Gender } \\
\hline Male & $8(44.44)$ & $44 \square 49.44 \square$ & $52 \square 48.6 \square$ \\
\hline Female & $10(55.56)$ & $45 \square 50.56 \square$ & $55 \square 51.4 \square$ \\
\hline \multicolumn{4}{|l|}{ Type of DM } \\
\hline Type I & $4(22.22)$ & $14 \square 15.73 \square$ & $18 \square 16.82 \square$ \\
\hline Type II & $14(77.78)$ & $75 \square 84.27 \square$ & $89 \square 83.18 \square$ \\
\hline \multicolumn{4}{|l|}{ HbA1c } \\
\hline$>9 \%$ & $14(77.78)$ & $68 \square 76.4 \square$ & $82 \square 76.64 \square$ \\
\hline$<=9 \%$ & $4(22.22)$ & $21 \llbracket 23.6 \square$ & $25 \square 23.36 \square$ \\
\hline \multicolumn{4}{|l|}{ DN } \\
\hline Yes & $11(61.11)$ & 14₫15.73凸 & $25 \square 23.36 \square$ \\
\hline No & $7(38.89)$ & 75凸84.27ロ & $82 \square 76.64 \square$ \\
\hline \multicolumn{4}{|l|}{ Hyperlipemia } \\
\hline Yes & $10(55.56)$ & $33 \llbracket 37.08 \square$ & $43 \llbracket 40.19 \square$ \\
\hline No & $8(44.44)$ & $56 \square 62.92 \square$ & 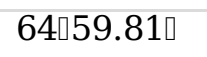 \\
\hline
\end{tabular}

\section{Cardiovascular diseases}

\begin{tabular}{cccc}
\hline Yes & $9(50)$ & $41 \square 46.07 \square$ & $50 \square 46.73 \square$ \\
No & $9(50)$ & $48 \square 53.93 \square$ & $57 \square 53.27 \square$ \\
Smoking & & & \\
Yes & $7(38.89)$ & $38 \square 42.7 \square$ & $45 \square 42.06 \square$ \\
\hline No & $11(61.11)$ & $51 \square 57.3 \square$ & $62 \square 57.94 \square$ \\
\hline Alcoholism & & & \\
\hline Yes & $11(61.11)$ & $48 \square 53.93 \square$ & $59 \square 55.14 \square$ \\
\hline No & $7(38.89)$ & $41 \square 46.07 \square$ & $48 \square 44.86 \square$ \\
\hline Anti-VEGF Therapy & & & \\
\hline Yes & $9(50)$ & $64 \square 71.91 \square$ & $73(68.22)$ \\
\hline No & $9(50)$ & $25 \square 28.09 \square$ & $34(31.78)$ \\
\hline History of PRP & & & \\
\hline Yes & $12(66.67)$ & $45 \square 50.56 \square$ & $57(53.27)$ \\
\hline No & $6(33.33)$ & $44 \llbracket 49.44 \square$ & $50(46.73)$ \\
\hline
\end{tabular}




\begin{tabular}{|c|c|c|c|}
\hline \multicolumn{4}{|l|}{ Cataract } \\
\hline Yes & $16(88.89)$ & $22 \llbracket 24.72 \square$ & $38(35.51)$ \\
\hline No & $2(11.11)$ & 67ロ75.28ロ & $69(64.49)$ \\
\hline \multicolumn{4}{|l|}{ Lens Status } \\
\hline Aphakia & $1(5.56)$ & $9 \square 10.11 \square$ & $10(9.35)$ \\
\hline Phakia & $2(11.11)$ & $25 \square 28.09 \square$ & $27(25.23)$ \\
\hline Pseudophakia & $15(83.33)$ & $55 \square 61.8 \square$ & $70(65.42)$ \\
\hline \multicolumn{4}{|c|}{ Vitreous tamponade } \\
\hline Silicone oil & $14(77.78)$ & $41 \square 46.07 \square$ & $55(51.4)$ \\
\hline Gas & $2(11.11)$ & $23 \llbracket 25.84 \square$ & $25(23.36)$ \\
\hline None & $2 \square 11.11 \square$ & $25 \square 28.09 \square$ & $27(25.24)$ \\
\hline \multicolumn{4}{|l|}{ ILM peeling } \\
\hline Yes & $12 \llbracket 66.67 \square$ & $43 \llbracket 48.31 \square$ & $55(51.4)$ \\
\hline No & $6 \square 33.33 \square$ & $46 \square 51.69 \square$ & $52(48.6)$ \\
\hline \multicolumn{4}{|l|}{ VH } \\
\hline Yes & $15 \square 83.33 \square$ & 48ロ53.93凸 & $63(58.88)$ \\
\hline No & $3 \square 16.67 \square$ & $41 \square 46.07 \square$ & $44(41.12)$ \\
\hline \multicolumn{4}{|l|}{ Tractional RD } \\
\hline Yes & $10 \square 55.56 \square$ & $45 \square 50.56 \square$ & $55(51.4)$ \\
\hline No & $8 \square 44.44 \square$ & $44 \llbracket 49.44 \square$ & $52(48.6)$ \\
\hline
\end{tabular}

\section{Detection of Associated factors of postoperative NVG in PDR patients}

Using the LASSO method, clinical features that might influence the prognosis were reduced in number.Summarized from medical records and surgical procedures, 3 potential predicators were selected from 17 features based on 107 patients in the cohort(Figure $a$ and $b$ ).in the LASSO regression model.Such included features are listed as follows:HbAlc,diabetic nephropathy(DN) and anti-VEGF therapy.

The results of the logistic regression analysis among HbAlc,diabetic nephropathy(DN) and anti-VEGF therapy are displayed in Table 2. HbAlc $(p<0.001)$ and diabetic nephropathy $(D N)(p<0.001)$ were selected

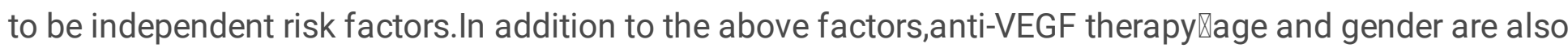
clinically meaningful factors which are incorporated in the nomogram(Figure c).

Table 2 Prediction factors for postoperative NVG in PDR patients.Note: $\beta$ is the regression coefficient. 


\begin{tabular}{|c|c|}
\hline \multirow[t]{2}{*}{ Intercept and variable } & Prediction model \\
\hline & Odds ratio (95\% CI) P-value \\
\hline Intercept & $-2.03140 .131(0.002-4.728) \quad 0.289$ \\
\hline HbA1c & $2.21389 .150(2.542-39.781)<0.001$ \\
\hline Diabetic nephropathy(DN) & $1.82496 .202(1.701-24.431)<0.001$ \\
\hline Anti-VEGF therapy & $-0.99660 .369(0.090-1.423)$ \\
\hline
\end{tabular}

\section{Internal Validation of the predicting model}

Internal validation was conducted to testify the predicting model,and related results are listed as follows. Firstly, the calibration curve of the NVG risk nomogram for the prediction of NVG risk in postoperative PDR patients demonstrated good consistency in this cohort(Figure d). Secondly,the C-index for the prediction nomogram was 0.852 (95\% Cl: $0.740-0.964)$ for the cohort, and was confirmed to be 0.8492 through bootstrapping validation,which also indicated that the predicted result was highly consistent with the actual observed result.In the NVG risk nomogram,it proves a good prediction capability.

\section{Clinical Utilization}

The decision curve analysis for the NVG nomogram is presented as follows(Figure e). The decision curve showed that if the threshold probability of a patient and a doctor is $>2 \%$, which means application of this nomogram is convincing and its clinical usefulness to help clinical decision-making has also been confirmed.

\section{Discussion}

Vitrectomy for proliferative diabetic retinopathy (PDR) has been reported to be associated with postoperative neovascular glaucoma (NVG) in $4 \%-12 \%$ of cases[13-15].In our study, $16.8 \%$ of PDR patients developed postoperative NVG, which is a bit higher than that percentage.A conceivable explanation of this phenomenon may be due to the different severity of underlying conditions in the patient group that were enrolled.

In the risk factor analysis, HbAlc,concomitant diabetic nephropathy(DN) and anti-VEGF therapy were associated with risk of postoperative NVG secondary to PDR. This nomogram suggested that low-level $\mathrm{HbAlc}(<9 \%)$,alleviation of DN and anti-VEGF therapy may be the key individual factors that could reduce the incidence of NVG.In a multicenter study conducted by Takayama and his colleagues[16],younger age, uncontrolled diabetes, no PRP, and postoperative $\mathrm{VH}$ were considered to be risk factors for postoperative NVG in PDR patients.Unconsistent with their study,neither age nor preoperative PRP has been screened out as prognostic factors for postoperative NVG in PDR patients.But our study have also found that highlevel HbAlc may indicate the occurrence of postoperative NVG.Microangiopathy caused by fluctuations in 
blood glucose levels has also been reported to contribute to the progression of ischemia[17].Previous publication highlighted that diabetic retinopathy is exacerbated when HbAlc is $6.5 \%$ or higher[18],the result of which correlates with our study.So to the best of knowledge,a better control of diabetes mellitus is the key for better prognosis.But a negative correlation between HbAlc and NVG onset was found by Masashi Sakamoto and his colleagues[19],where they ascribed the contradictory result to excessive administration of insulin in patients with NVG and the lack of investigation in the course of diabetic retinopathy.So further studies are still needed to observe the relationship between $\mathrm{HbA1c}$ level and NVG onset in PDR patients.

Selected by lasso regression model,diabetic nephropathy(DN) may be a significant risk factor of postoperative NVG onset.As previously reported,diabetic retionopathy has been associated with a faster decline in the estimated glomerular filtration rate(eGFR) among the general population and the elderly population with or without diabetes[20-21].Chi-Chih Hung[22] and his colleagues have also found that diabetic retinopathy was significantly associated with an increased risk for end-stage renal disease.In our study, DN could also be considered as an important risk factor for NVG onset to some extent.In addition,some systematic abnormalities,such as cardiovascular diseases, have been found to be entwined with postoperative NVG in PDR patients.

It could not be overemphasized that VEGF is a pivotal angiogenic factor induced by retinal ischemia,and the vitreous level of VEGF at the time of vitrectomy is associated with a significant risk of both the postoperative progression of PDR and the occurrence of postoperative complications[23-24].Anti-VEGF therapy has been validated to be effective in promoting the absorption of $\mathrm{VH}$ and visual rehabilitation.So in the management of PDR, it is important to control ischemic stimuli primarily by anti-VEGF therapy as a preoperative therapy,which is combined with other methods,such as panretinal photocoagulation and vitrectomy.However,Wakabayashi et al[25] did not find significant relation between vitreous VEGF levels at the time of vitrectomy and postoperative late complications due to persistent overproduction of VEGF after surgery,which reminded us that it is essential to continuously detect and suppress the level of VEGF.We speculated that persistent secretion of VEGF might ascribe to underlying comorbidities that might exacerbate retinal ischemia.

Current study still have some limitations.Firstly,this nomogram requires external validation, and further research is needed to observe the risk and prognostic factors related to postoperative NVG onset.Secondly, the possibility of selection bias with respect to severity of the disease has to be considered in interpreting the results of this study.Thirdly,the retrospective nature.

\section{Conclusion}

In conclusion ,a novel nomogram with a relatively good accuracy has already been established for ophthalmologists to access the risk of postoperative NVG onset in PDR patients. In a nutshell,its clinical usefulness has also been testified by calibration plot and C-index, which is intended for helping ophthalmologists to take interventions in time,and avoid delays in treatment when there is a high 
probability of postoperative NVG.More importantly,taking good control of HbAlc level,awaring of diabetic nephropathy and other underlying comorbidities,continuous detecting and suppressing the VEGF level are important interventions for avoiding postoperative NVG secondary to PDR.

\section{Abbreviations}

NVG:neovascular glaucoma

PDR:proliferative diabetic retinopathy

IOP: intraocular pressure

VEGF:anti-vascular endothelial growth factor

PRP:panretinal photocoagulation

LASSO:the least absolute shrinkage and selection operator

SE:standard error

DN:diabetic nephropathy

DM:diabetic mellitus

HbAlc:Glycosylated Hemoglobin

ILM:internal limiting membrane

OR: odds ratio

Cl:confidence interval

VH:vitreous hemorrhage

RD: retinal detachment

eGFR: estimated glomerular filtration rate

\section{Declarations}

\section{Ethics approval and Consent to participate:}

This study followed the tenets of the Declarations of Helsinki and was approved by the ethical committee of Shenyang Fourth People's Hospital. Written consent to participate were obtained from all the patients.

\section{Consent to publish:}


All the patients agreed to the publication of this article and related clinical data.

\section{Availability of data and material}

The data that support the findings of this study are available from the electronic records of Shenyang Fourth People's Hospital,but restrictions apply to the availability of these data, which were used under license for the current study, and so are not publicly available. Data are however available from the authors upon reasonable request and with permission of Shenyang Fourth People's Hospital.

\section{Competing Interests:}

The authors have no conflicts of interest to declare.

\section{Funding:}

No Funding

\section{Authors' Contributions $\square$}

$\mathrm{LX}$ and $\mathrm{XLC}$ collected the data,XYZ drafted the article, $\mathrm{XX}, \mathrm{XLC}$ and $\mathrm{XYZ}$ carried out critical revision and correction of the manuscript.All authors read and approved the final manuscript.

\section{Acknowledgements:}

Not Applicable

\section{References}

1. Havens SJ, Gulati V.Neovascular glaucoma.Dev Ophthalmolø2016,55:196-204

2. Simha A, Aziz K, Braganza A et al.Anti-vascular endothelial growth factor for neovascular glaucoma. Cochrane Database of Systematic Reviews,2020区 Issue 2. Art. No.: CD007920. DOI: 10.1002/14651858.CD007920.pub3

3. Sauerbrei W, Royston P, Binder H. Selection of important variables and determination of functional form for continuous predictors in multivariable model building. Stat Med.2007囚 26(30):5512-5528

4. Friedman J, Hastie T, Tibshirani R. Regularization paths for generalized linear models via coordinate descent. J Stat Softw. 2010®33(1):1-22

5. Kidd AC, McGettrick M, Tsim S, Halligan DL, Bylesjo M, Blyth KG.Survival prediction in mesothelioma using a scalable LASSO regression model: instructions for use and initial performance using clinical predictors. BMJ Open Respir Res.2018ه5(1):e000240.

6. Xing J, Min L, Zhu S, et al. Factors associated with gastric adenocarcinoma and dysplasia in patients with chronic gastritis: a population-based study. Chin J Cancer Res.2017区29(4):341-350 
7. Balachandran VP, Gonen M, Smith JJ, DeMatteo RP. Nomograms in oncology: more than meets the eye. Lancet Oncol.2015囚16(4):e173-e180.

8. lasonos A, Schrag D, Raj GV, Panageas KS. How to build and interpret a nomogram for cancer prognosis. J Clin Oncol. 2008®26(8):1364-1370.

9. Kramer AA, Zimmerman JE. Assessing the calibration of mortality benchmarks in critical care: the Hosmer-Lemeshow test revisited. Crit Care Med.2007ه35(9):2052-2056.

10. Pencina MJ, D'Agostino RB. Overall $C$ as a measure of discrimination in survival analysis: model specific population value and confidence interval estimation.Stat Med. 2004囚23(13):2109-2123.

11. Vickers AJ, Cronin AM, Elkin EB, Gonen M. Extensions to decision curve analysis, a novel method for evaluating diagnostic tests, prediction models and molecular markers. BMC Med Inform Decis Mak.2008ه8:53.

12. Huang $\mathrm{YQ}$, Liang $\mathrm{CH}, \mathrm{He} \mathrm{L}$, et al. Development and validation of a radiomics nomogram for preoperative prediction of lymph node metastasis in colorectal cancer.J Clin Oncol.2016》 34(18):2157-2164.

13. Goto A, Inatani $M$, Inoue $T$, et al. Frequency and risk factors for neovascular glaucoma after vitrectomy in eyes with proliferative diabetic retinopathy. J Glaucoma. 2013ヌ22:572-6

14. Huang $\mathrm{CH}$, Hsieh $\mathrm{YT}$, Yang $\mathrm{CM}$. Vitrectomy for complications of proliferative diabetic retinopathy in young adults: clinical features and surgical outcomes. Graefes Arch Clin Exp Ophthalmol. 2017区 255:863-871

15. Xu Liang,Yue Zhang,Yu-Peng Li et al.Frequency and Risk Factors for Neovascular Glaucoma after Vitrectomy in Eyes with Diabetic Retinopathy:An Observational Study.Diabetes Ther,2019『 https://doi.org/10.1007/s13300-019-0644-0

16. Kei Takayama, Hideaki Someya, HiroshiYokoyama,et al.Risk Factors of Neovascular Glaucoma After 25-gauge Vitrectomy for Proliferative Diabetic Retinopathy with Vitreous Hemorrhage: A Retrospective Multicenter Study.Sci Rep,2019『9:14858.Doi:https://doi.org/10.1038/s41598-01951411-6

17. Osaadon P,Fagan XJ,Lifshitz T et al.A review of anti-VEGF agents for proliferative diabetic retinopathy.Eye(Lond),2014, 28(5): 510-520. DOI: 10.1038/eye.2014.13.

18. Lachin JM, Bebu I, Bergenstal RM, et al. Association of glycemic variability in type 1 diabetes with progression of microvascular outcomes in the diabetes control and complications trial. Diabetes Care.2017, 40(6):777-783

19. Jin P, Peng J, Zou H, et al.A five-year prospective study of diabetic retinopathy progression in Chinese type 2 diabetes patients with "well-controlled" blood glucose. PLoS One. 2015,10(4):e0123449

20. Masashi Sakamoto,Ryuya Hashimoto,Izumi Yoshida et al.Risk factors for neovascular glaucoma after vitrectomy in eyes with proliferative diabetic retinopathy.Clinical Ophthalmology ,2016,12 2323-2329 
21. Wong, T. Y. et al. Retinal microvascular abnormalities and renal dysfunction: the atherosclerosis risk in communities study. Journal of the American Society of Nephrology: JASN, 2014,15, 2469-2476, doi:10.1097/01.ASN.0000136133.28194.E4.

22. Edwards, M. S. et al. Associations between retinal microvascular abnormalities and declining renal function in the elderly population: the Cardiovascular Health Study. American journal of kidney diseases: the official journal of the National Kidney Foundation,2005,46, 214-224, doi:10.1053/j.ajkd.2005.05.005.

23. Chi-Chih Hung, Hugo You-Hsien Lin, Daw-Yang Hwang et al.Diabetic Retinopathy and Clinical Parameters Favoring the Presence of Diabetic Nephropathy could Predict Renal Outcome in Patients with Diabetic Kidney Disease.Scientific Reports,2017,7,1236.DOI:10.1038/s41598-017-01204-6

24. J. Wang, S. Chen, F. Jiang et al.“Vitreous and plasma VEGFlevels as predictive factors in the progression of proliferativediabetic retinopathy after vitrectomy,"PLoS One, 2014,vol. 9,no. 10, Article ID e110531

25. Y. Suzuki, K. Suzuki, T. Kudo, T. Metoki, and M. Nakazawa."Level of vascular endothelial growth factor in the vitreous fluid of proliferative diabetic retinopathy patients and prognosis after vitrectomy," Ophthalmologica, 2016,vol. 236, no. 3,133-138

26. Y. Wakabayashi, Y. Usui, K. Tsubota et al. "Persistent overproduction of intraocular vascular endothelial growth factor as a cause of late vitreous hemorrhage after vitrectomy for proliferative diabetic retinopathy," Retina,2017,vol. 37, no. 12,2317-2325.

\section{Figures}




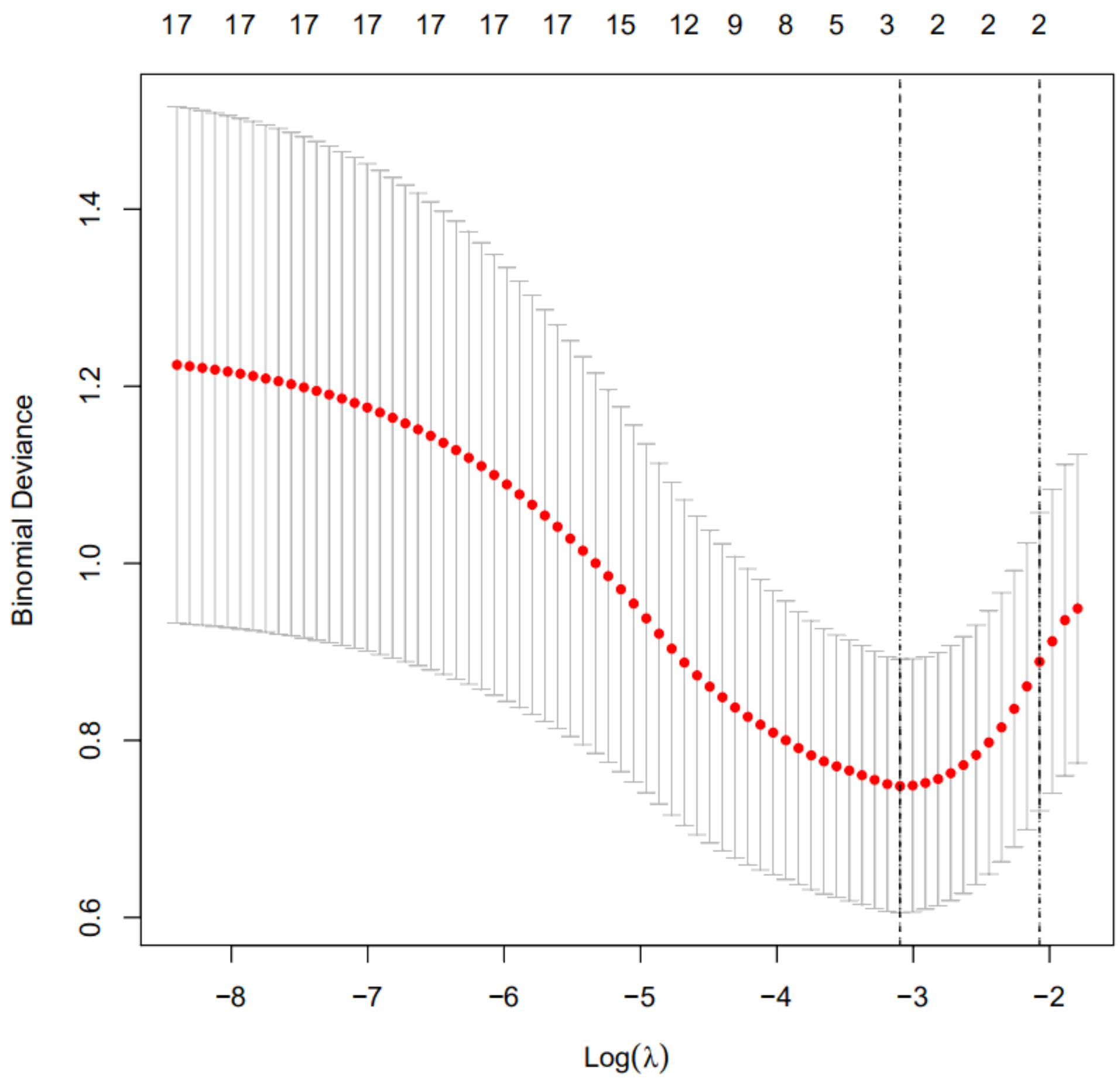

Figure 1

Demographic and clinical feature selection using the LASSO binary logistic regression model. Notes: (a) Optimal parameter (lambda) selection in the LASSO model used five fold cross-validation via minimum criteria. The partial likelihood deviance (binomial deviance) curve was plotted versus log(lambda). Dotted vertical lines were drawn at the optimal values by using the minimum criteria and the $1 \mathrm{SE}$ of the minimum criteria (the 1-SE criteria). 


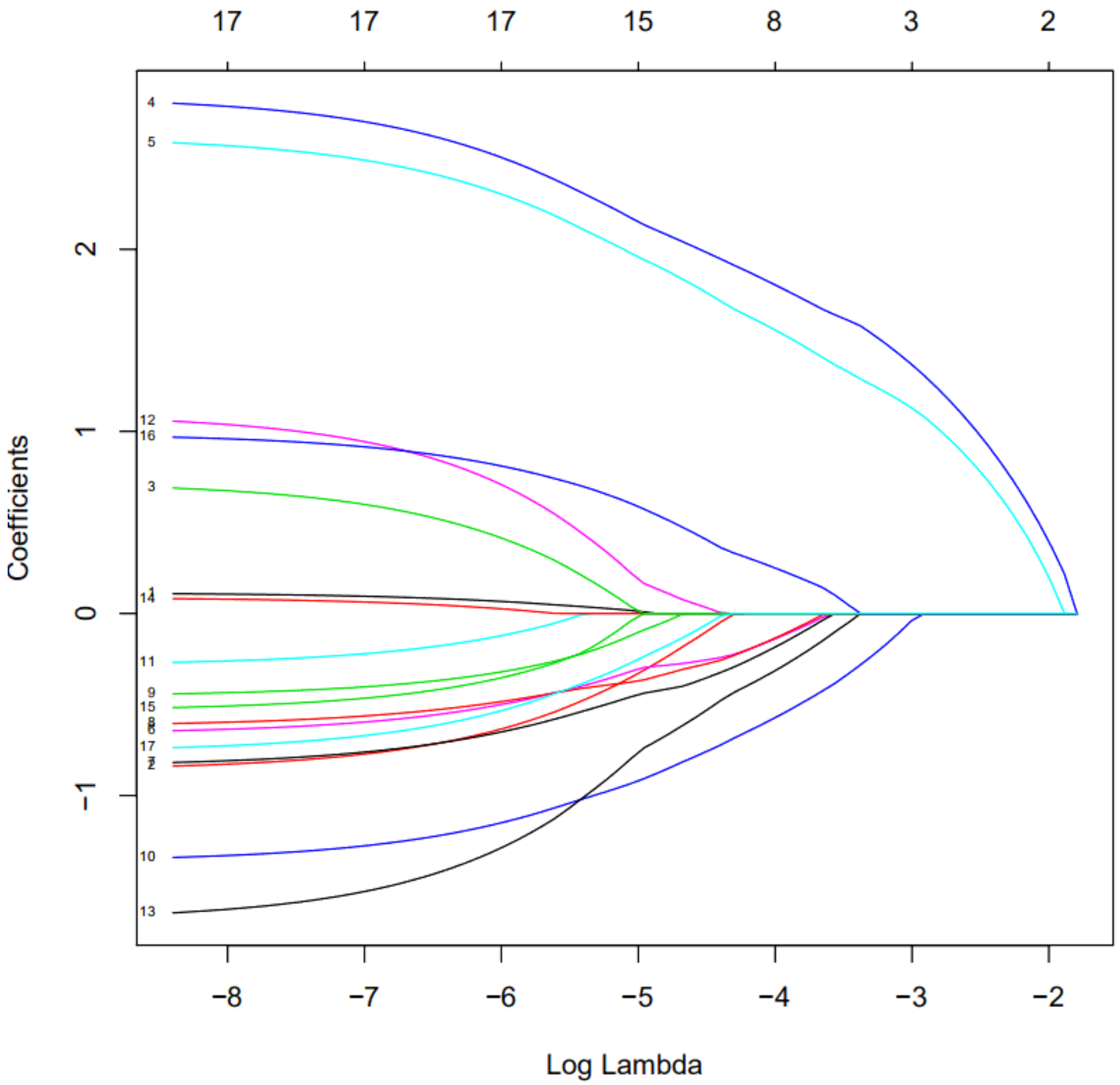

Figure 2

Demographic and clinical feature selection using the LASSO binary logistic regression model. (b) LASSO coefficient profiles of the 17 features. A coefficient profile plot was produced against the log(lambda) sequence. Vertical line was drawn at the value selected using fivefold cross-validation, where optimal lambda resulted in three features with nonzero coefficients. 
Points

$\begin{array}{lllllllllll}0 & 10 & 20 & 30 & 40 & 50 & 60 & 70 & 80 & 90 & 100\end{array}$

$\mathrm{HbA1c}$

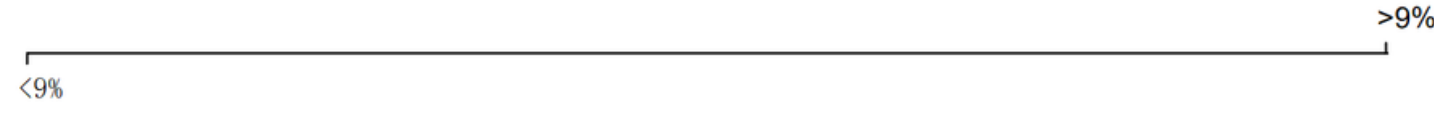

DN

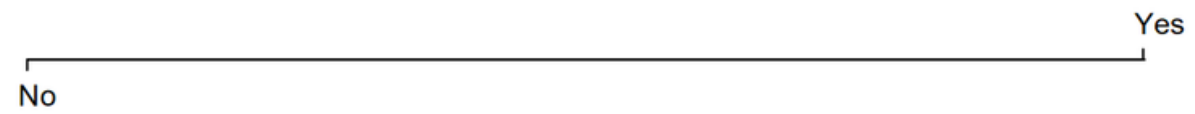

anti-VEGF

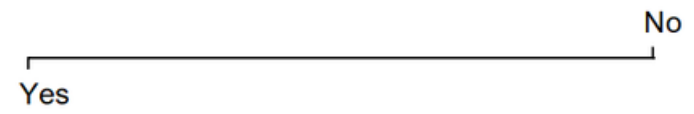

Gender

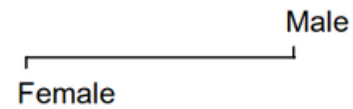

Age

60-69

$\stackrel{\mathrm{T}}{>=70}$

Total Points

\begin{tabular}{lllllllllllllll}
\hline & 20 & 40 & 60 & 80 & 100 & 120 & 140 & 160 & 180 & 200 & 220 & 240 & 260 & 280
\end{tabular}

Risk of NVG

\begin{tabular}{llllllll}
\hline 0.1 & 0.2 & 0.3 & 0.4 & 0.5 & 0.6 & 0.7 & 0.8
\end{tabular}

\section{Figure 3}

Developed postoperative NVG nomogram. Note: The postoperative NVG nomogram was developed in the cohort, with HbA1c level, DN, anti-VEGF therapy,Age,Gender incorporated. 


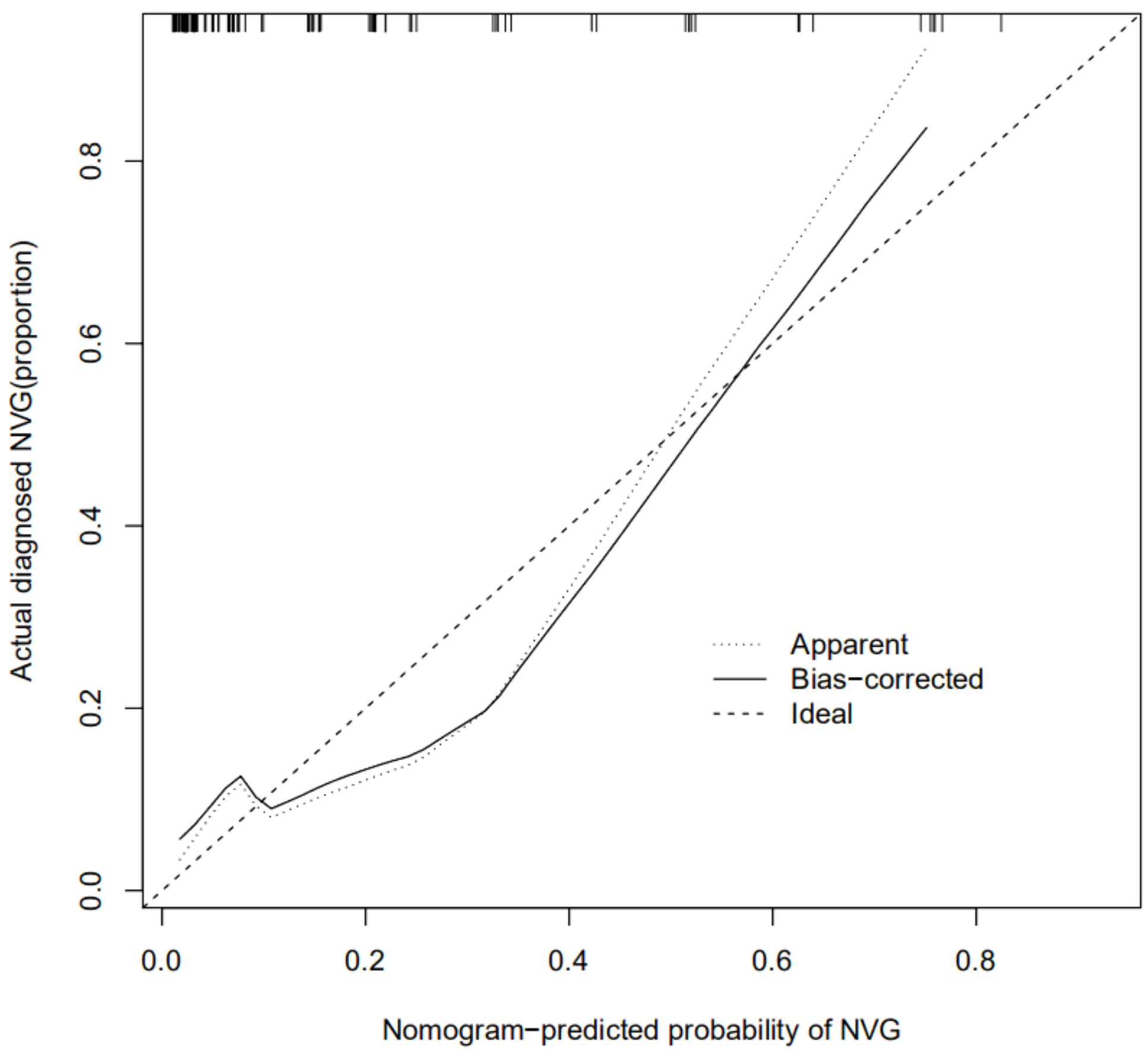

Figure 4

Calibration curves of the NVG nomogram prediction in the cohort. Notes: The x-axis represents the predicted postoperative NVG risk. The y-axis represents the actual postoperative NVG in PDR patients. The diagonal dotted line represents a perfect prediction by an ideal model. The solid line represents the performance of the nomogram, of which a closer fit to the diagonal dotted line represents a better prediction. 


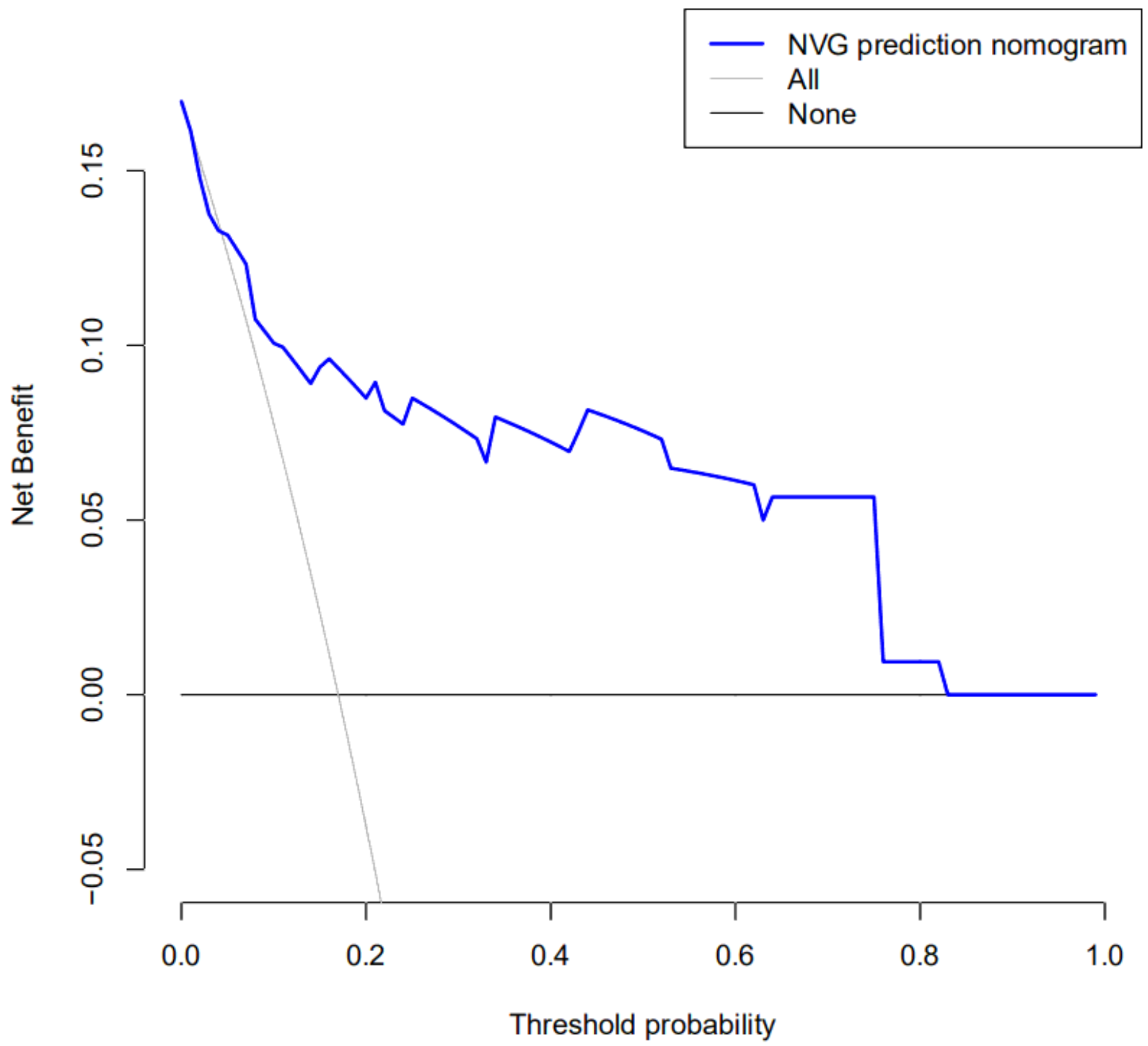

Figure 5

Decision curve analysis for the postoperative NVG nomogram. Notes: The y-axis measures the net benefit. The dotted line represents the NVG risk nomogram. The thin solid line represents the assumption that all patients presented with postoperative NVG. Thin thick solid line represents the assumption that no patients presented with postoperative NVG. The decision curve showed that if the threshold probability of a patient and a doctor is $2 \%$ and $100 \%$, respectively, using this NVG nomogram in the current study to predict postoperative risk adds more benefit than the intervention-all-patients scheme or the interventionnone scheme. 